\title{
A Review of Volt Var Optimization Techniques
}

\section{Mahendru $\mathrm{A}^{1 *}$ and Deshpande $\mathrm{P}^{2}$}

${ }^{1} E C E$ Department, Western University, Ontario, Canada

${ }^{2} E C E$ Department, University of Ottawa, Canada

\begin{abstract}
Volt Var Optimization (VVO) and Conservation Voltage Reduction (CVR) provide an opportunity to improve the power system performance in terms of efficiency and usability. Many papers have been written on different algorithms and methodologies to implement these technologies. This paper aims to review the important developments in this field in the form of review of some of the existing papers. The technology review is followed by discussion on the scope for further research and improvements in this field.
\end{abstract}

Keywords: Volt Var optimization; Conservation voltage reduction; Multi objective optimization; Distribution system; Voltage reduction

\section{Introduction}

The power utilities around the globe are always looking for solutions to improve or maintain the power quality and efficiently operate their distribution systems without considerable capital expenditure. These requirements have resulted in evolution of technologies like Volt Var optimization (VVO) and Conservation Voltage Reduction (CVR). Traditional Voltage/VAR management technologies have been used by the power industry for over 30 years to reduce electric line losses and increase grid efficiency [1]. However, interest in CVR technique is increasing drastically because of rapid development in the field of smart grid technologies $[2,3]$.

A review of some important papers has been done to develop an overview of the current VVO technology and scope for future research work. In each of the review, the mathematical models developed by the authors have been discussed along with the simulation results and any limitations to highlight future area of research that could result in improvements in the current technology.

\section{Background}

Whenever there is a flow of power from a circuit element, it results in voltage drop across the element. The amount of voltage drop varies with the amount of power flowing through the element. This makes the voltages in a distribution system variable and hence a need to control them to maintain voltages within a specified range. If the circuit element has inductive impedance, it causes a phase lag between the voltage and current phasor. Thus, to deliver same amount of real power more current needs to flow through the system as compared to pure resistive impedance. Figure 1 shows a typical voltage profile along a distribution line [4]. The whole concept of VVO and CVR is to maintain these voltages and the phase lag within certain limits or specified values by using tap changers or by reactive power injection. These voltage limits or specific voltages at various locations in the distribution system are calculated using optimization of the mathematical models of the system.

\section{Concept of VVO and CVR}

The principle of CVR is that savings in energy consumption is achieved by a reduction of voltage level without affecting the performance of the customer's devices demand and minimize the system losses (engine). This means that total power demand can be reduced by operating in the lower allowable range of the voltage as shown in Figure 2 [4].
Every power system requires a combination of real power and reactive power (due to phase lag as mentioned earlier). The currents caused by the reactive power flow, flows through the lines and contribute towards resistive losses in the system. Thus, utilities try to reduce these losses by injecting local reactive power into the system near the loads that in turn reduces the current flowing in the lines due

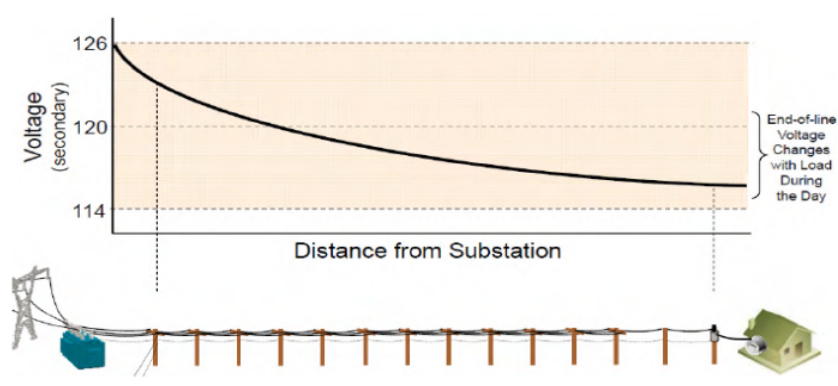

Figure 1: Voltage profile along a typical distribution line.

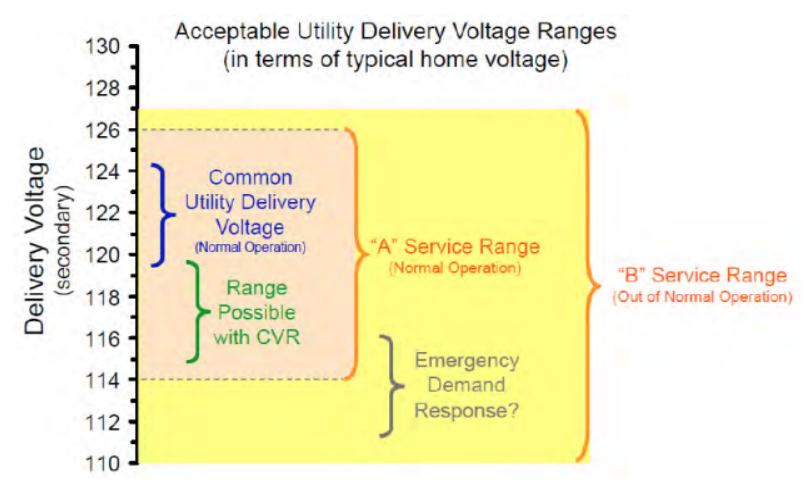

Figure 2: ANSI C84.1 Service Voltage Levels.

*Corresponding author: Mahendru A, ECE Department, Western University 1151, Richmond Street, London, Ontario N6A 3K7, Canada, Tel: +12262687454 E-mail: mahendruankush@gmail.com

Received July 03, 2018; Accepted July 27, 2018; Published August 03, 2018

Citation: Mahendru A, Deshpande P (2018) A Review of Volt Var Optimization Techniques. J Electr Electron Syst 7: 265. doi: 10.4172/2332-0796.1000265

Copyright: (c 2018 Mahendru A, et al. This is an open-access article distributed under the terms of the Creative Commons Attribution License, which permits unrestricted use, distribution, and reproduction in any medium, provided the original author and source are credited. 
to reactive power flow. This is referred to as VAR control. But, injecting reactive power affects the system voltages. This concept of optimizing the system losses by reducing reactive power flow which in turn affects system voltages is referred to as Volt Var Optimization. Figure 3 shows a smart optimization framework proposed by Singh S et al. [5].

\section{Concept of multi objective optimization}

The problem of optimizing a distribution system involves multi objectives i.e. minimizing the losses and reducing the overall power demand. Thus, often the systems are modeled as multi objective optimization problems ie having more than one objective functions. Now an optimum solution for one objective function may not be optimum for other objective function. Therefore, concept of pareto optimum solutions is used to solve such problems. A set of pareto optimum solutions are the set of solution such that no other solution would result in better solution of any one of the objective functions without compromising with the values of other objective functions.

\section{Concepts of formulating optimization problem}

Formulation of an optimization problem means mathematical representation of the system in terms of objective functions and constraints. The objective functions are typically system losses and total power consumption but may differ according to the variable to be optimized. The constraints set consists of the system voltage limits, power flow equations, power factor limits and load ability limit of the individual lines. Different methods are employed for solving these optimization problems that include genetic algorithms, linear programs, mixed integer quadratically constraint program. The main factors which generally decide the selection of right optimization methods are depending upon nature and number of objective function, constraints and inner behavior of the control variables [5].

\section{Review of Research}

The following section presents a review of papers [6-10]

\section{Multi objective optimization to peak load relief \& energy efficiency}

General overview and contribution: This paper aims to solve

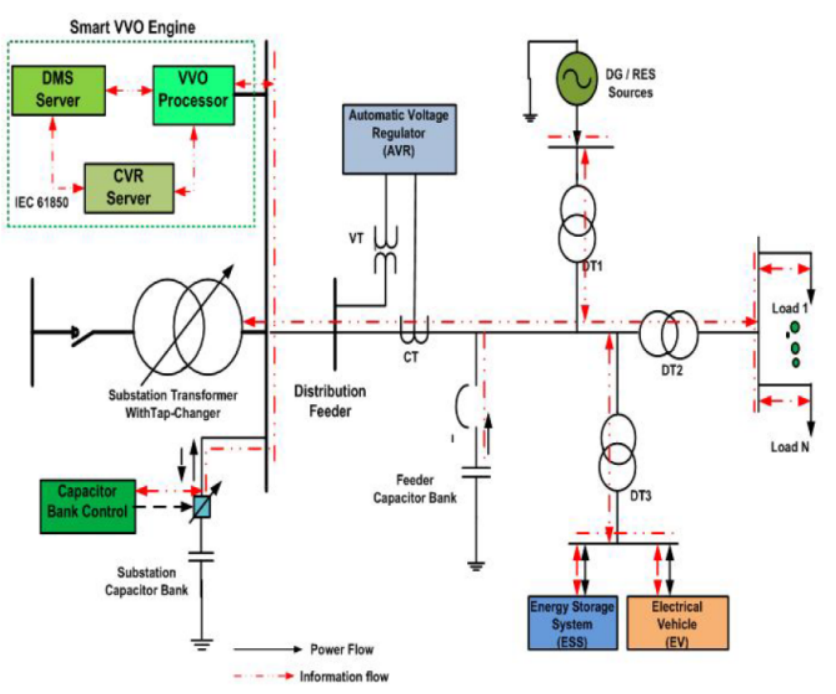

Figure 3: Illustration of a smart VVO framework the VVO problem considering CVR as a part of the problem using multi objective optimization using genetic algorithm (NSGA-II) [11] The genetic algorithm used gives a set of solutions which can be used according to the user requirement [6].

To consider, the variation of the load with voltage, the author used the exponential load model as shown in equation (1) and (2).

$$
\begin{gathered}
P l_{i}=P_{n, i}\left(\frac{V_{i}}{V_{n}}\right)^{k_{p}} \\
Q l_{i}=Q_{n, i}\left(\frac{V_{i}}{V_{n}}\right)^{k_{q}}
\end{gathered}
$$

Where $P l_{\mathrm{i}} / Q_{l_{i}}$ and $P_{\mathrm{n}^{\prime} i} / Q_{n^{\prime} i}$ denotes active/reactive load power and active load power at nominal voltage at bus i respectively. $V_{i}$ represents voltage at bus $i$ and $V_{n}$ represents nominal voltage. The values of the exponents were found [12].

The first function to be minimized is the total power drawn by the distribution system form the substation. It consists of the load power and the line losses which are represented as;

$$
\begin{aligned}
& f_{1}=P_{\text {in }}=P_{\text {load }}+P_{\text {loss }} \\
& P_{\text {load }}=\sum_{i \in n b} P l_{i}\left(V_{i}\right) \\
& P_{\text {load }}=\sum_{i \in n b} \sum_{i \in n b} \Delta V_{i j}^{2} \frac{r_{i j}}{Z_{i j}^{2}}
\end{aligned}
$$

Here $P_{\text {load }}$ and $P_{\text {loss }}$ represents total active load power and total losses. $n b$ is the set of bus and $\mathrm{i}, \mathrm{j}$ are indices for bus number. $\mathrm{r}_{\mathrm{ij}}$ and $\mathrm{Z}_{\mathrm{i}}$ represents resistance and impedance between bus $i$ and $j$ respectively. The second function to be minimized is the voltage deviation from the nominal value which can be represented by;

$$
\begin{aligned}
& f_{2}=\frac{1}{n b} \sum_{i \in n b}\left(1-\mu_{i}\right) \\
& \mu_{i}=\left\{\begin{array}{l}
\frac{V_{i}-V_{\text {min }}}{V_{n}-V_{\text {min }}} \\
\frac{V_{\max }-V_{i}}{V_{\text {max }}-V_{n}}
\end{array}\right.
\end{aligned}
$$

Where $V_{\min }$ and $V_{\max }$ are minimum and maximum values of voltage according to the standard followed. The minimization of the objective function (6) signifies that the best value of the voltages at each bus is the nominal value. The constraints consist of the following set of equations

$$
\begin{aligned}
& P g_{i}-P l_{i}-P_{i}(\theta, V, \text { tap })=0 \\
& Q g_{i}-Q l_{i}+Q C_{i}-Q_{i}(\theta, V, \text { tap })=0 \\
& V_{\min } V_{i} \leq V_{\max } \\
& p f_{\min } \leq p f_{\text {sys }} \leq p f_{\max } \\
& R G_{i}=1-\operatorname{tap}_{i} \Delta t a p_{i} \\
& C_{i}^{s w}=n s w_{i} \Delta C_{i}
\end{aligned}
$$

Where $P g_{i} / \mathrm{Qg}_{\mathrm{i}}$ is the active/reactive power generation at bus $\mathrm{i}, P_{\mathrm{i}} /$ $\mathrm{Q}_{\mathrm{i}}$ is the active/reactive power injection at bus $\mathrm{i}, \mathrm{QC}_{\mathrm{i}}$ is the reactive power injection by capacitor at bus $\mathrm{i}, R G_{i}$ is the regulation ratio at bus $\mathrm{i}, C_{i}^{\mathrm{sw}}$ is the reactive power injection by switched capacitor at bus $\mathrm{i}$, $\operatorname{tap}_{\mathrm{i}}$ and $\Delta \operatorname{tap}_{i}$ are the selected tap of the device and the step of voltage variation of the device at bus $\mathrm{i}, n s w_{\mathrm{i}}$ and $\Delta C_{i}$ are the selected position 
of the switched capacitor and the step of reactive power variation of the capacitor. Here equations (8), (9), (10), (11), (12) and (13) represent the active power balance, reactive power balance, voltage range limits, discrete transformer tap changer ratio and the discrete steps of shunt connected capacitors respectively.

The above formulated problem is then solved using Elitist Non Dominated Sorting Genetic Algorithm -II (NSGA-II) The initial solution is started with randomly generated pareto solutions. Tournament selection, crossover, and mutation are applied to obtain an offspring population of size $N$ [2]. The process is repeated till a fixed number of iterations are reached.

The results were taken from the UKGDS-95 buses system [14], and the IEEE 34-bus feeder [15]. Only results based on UKGDS-95 system are presented in this paper. The distribution system schematic is shown in Figure 4. The voltage and power factor limits used were 0.95 p.u. to 1.05 p.u. and 0.96 lag to 0.99 lag respectively. The control devices used, their specifications and the exponent values for the load model are mentioned in (paper 2). Figure 5 shows a set of non-dominated solutions for peak load at 19:00 hours. The maximum energy saving solution represents solution that is most favourable to the objective function $f_{2}$ and least favourable to the objective function $f_{1}$ while solution marked moderate energy savings represent a balanced solution for both the objective functions. Figure 6 shows the reduction in hourly power intake of the system for the above two solutions discussed and

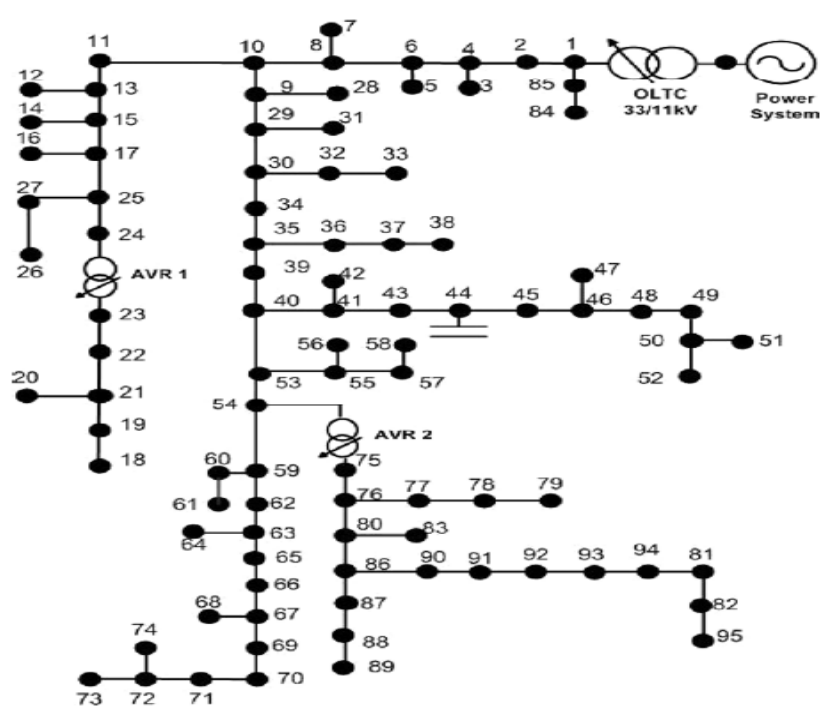

Figure 4: UKGDS-95 buses system used for simulation.

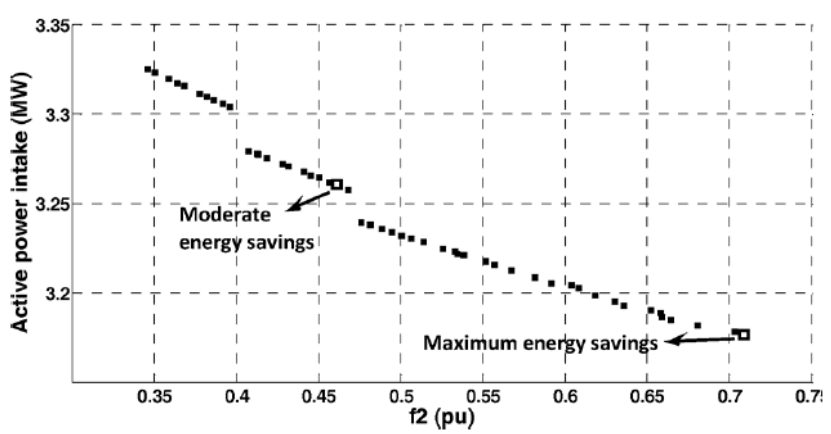

Figure 5: Non dominated solutions obtained at 19:00

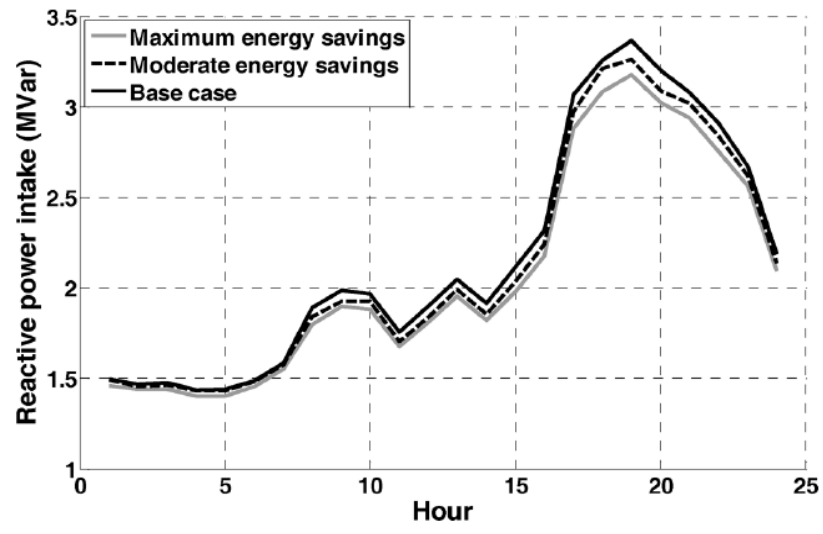

Figures 6: Active power intake profiles.

the base case. The results show that there is $2.28 \%$ and $4.55 \%$ reduction in case of moderate and maximum energy savings solution respectively.

Advantages: This paper introduces a genetic algorithm to solve the optimization problem without making any assumptions in terms of mathematical modelling. The solution also offers flexibility with respect to the solution implementation according to the choice of the user. The effectiveness can be seen form the results obtained.

Drawbacks and scope for improvement: The second objective function $\mathrm{f}_{2}$ minimizes the total voltage deviation from nominal value in the system. This objective does not take into account the opportunity to obtain even lower levels of power consumption by operating in lower range of the specified voltage range (CVR). Incorporating this into the objective function could have obtained different results. The author presented this solution as a solution to day ahead forecast data instead of real time implementation. Due to inaccuracies of the forecast the solutions obtained will not remain optimal in real implementation.

The problem also does not consider the number of switching actions of the control devices.

\section{On multiobjective Volt-Var optimization in power systems}

General overview and contribution: This paper [7] also deals with multi objective optimization using genetic algorithms. But, aims to solve the problem of reactive power allocation in the planning stage to minimize the initial investment and obtain maximum benefit. It solves the distribution system and transmission system separately and using an algorithm, combines them to obtain the optimum solution.

The mathematical model of the distribution system is represented by following equations:

$$
\begin{aligned}
& \text { Minimize: } \mathrm{P}_{\text {loss }}=\mathrm{P}_{\text {loss }}\left(\delta, \mathrm{V}, \mathrm{Q}_{\mathrm{c}}\right) \\
& \text { Minimize: } \sum_{i=1}^{11} Q_{c i} \cdot P_{F} \\
& \text { Subject to: } \boldsymbol{G}\left(\boldsymbol{\delta}, \boldsymbol{V}, \boldsymbol{Q}_{C}\right)=0 \\
& \mathrm{Q}_{\mathrm{ci}}=k Q_{c 0} \quad k=0,1,2, \ldots
\end{aligned}
$$

$Q_{c i}$ is the reactive support applied to bus I, $P_{F}$ is the price of per unit reactive power on the feeder and $Q_{c 0}$ is the incremental reactive power step. The first objective function represents the system losses and the second objective function represents the total investment. The constraints set consist of normal power flow equations and the voltage 
limits and the discrete nature of the injected reactive power. The transmission system was also represented by similar equations but with different cost per reactive power injected. The combined mathematical model was represented by following equations:

$$
\begin{aligned}
& \text { Minimize: } \Sigma \mathrm{P}_{\text {loss }}=\mathrm{P}_{\text {loss }, \mathrm{TS}}+\Sigma \mathrm{P}_{\text {loss } \mathrm{F}} \\
& \text { Subject to: } \boldsymbol{G}\left(\boldsymbol{\delta}, \boldsymbol{V}, \boldsymbol{Q}_{C}\right)=0 ; \\
& Q_{c i}=k Q_{c o T} \\
& Q_{c i}=k Q_{c o F} \\
& P_{T} \cdot \sum_{i} Q_{c i}+P_{F} \cdot \sum_{j} Q_{c j} \leq I . R .
\end{aligned}
$$

where $\mathrm{P}_{\text {loss,TS }}$ and $\mathrm{P}_{\text {loss }, \mathrm{F}}$ represent power loss in transmission line and distribution feeder respectively, $Q_{c j}$ is the reactive support applied at feeder $\mathrm{j}, Q_{c 0 T}$ is the transmission reactive support incremental step $Q_{c O F}, P_{T}$ is the price per unit reactive support in the transmission system and $I . R$. is the maximum investment resources. The objective function represents the combined distribution and transmission losses.

The algorithm explained in Bigovic MM et al. [7] is proposed to solve the combined problem of reactive resources allocation. The algorithm starts with maximum investment in the transmission system and uses sensitivity analysis to transfer the resources form least sensitive bus to the distribution system. The results obtained from this proposed method simulated on a test system suggest that the overall losses are minimized if, for the distribution system the pareto solution corresponding to maximum investment and minimum losses was used and for transmission system pareto solution corresponding to lower investment was combined. In other words, the algorithm suggested to transfer the reactive resources from transmission system to the distribution system. This solution was also verified using voltage stability analysis done by the authors.

Advantages: As the proposed method uses genetic algorithm, thus the actual system equations were used to solve the problem without any approximation. The proposed method provides a good optimized solution when planning investments in a small system. It is extremely useful if a utility has fixed amount of resources and there is a strict limit on the investments that can be made.

Drawbacks and scope for improvement: The proposed method does not consider the presence of tap changer transformers in the system. A large system may have many tap changers that reduces the requirement of reactive power injection.

It proposed solution is limited in the sense that it considers a fixed investment available and does not provide the incremental cost of investment in loss reduction so that a utility can also plan the amount of investment accordingly.

\section{A Framework for Volt-Var optimization in distributed system}

General overview and contribution: The paper [8] formulates the VVO problem as Mixed Integer Quadratically Constrained Programming (MIQCP) problem and uses branch and cut algorithm to solve the problem. It is also based on day ahead planning using load forecast. For a particular load conditions, only one optimal solution is obtained unlike the multi objective problems discussed in above papers. The ZIP load model is used (16), and the parameters are obtained by studying a typical residential area. The author categorized the main loads of a residential area in a cold climate and developed two types of homes having different combination of these loads which is shown in Table 1. Thus, a combination of these homes, street light loads and typical commercial loads around a residential area are connected to the distribution transformer for simulation purpose. The transformer core losses are represented as voltage dependent load connected in parallel. The behaviour of this combination of loads is studied to calculate the parameters of the ZIP load model.

$$
\begin{aligned}
& \frac{P(V)}{P_{0}}=C_{Z}\left(\frac{V}{V_{0}}\right)^{2}+C_{I}\left(\frac{V}{V_{0}}\right) \\
& \frac{Q(V)}{Q_{0}}=C_{Z}^{\prime}\left(\frac{V}{V_{0}}\right)^{2}+C_{I}^{\prime}\left(\frac{V}{V_{0}}\right)
\end{aligned}
$$

$P$ and $Q$ are load's active and reactive power consumption respectively, $V$ is the terminal voltage and subscript zero represents the nominal value. The objective functions are described by the following two equations;

$$
\begin{aligned}
& P_{\text {loss }}=\sum_{m<k} \bar{G}_{m, k}\left[\left(V_{m}^{r e}-V_{k}^{r e}\right)^{2}+\left(V_{m}^{i m}-V_{k}^{i m}\right)^{2}\right. \\
& P_{S}=-V_{S} \sum_{k=1}^{n}\left(\bar{G}_{S, k} V_{k}^{r e}-\bar{B}_{S, k} V_{k}^{i m}\right)
\end{aligned}
$$

Where $G$ and $B$ are real and imaginary parts of admittance matrix, $n$ is the number of nodes, $m$ and $k$ are at the two ends of the branch connecting node $m$ to node $k$. $V^{\text {re }}$ and $V^{i m}$ are the real and imaginary parts of the nodal voltages. $P$ is the active power drawn from the substation. Equation (18) is used as the objective function for the problem when the objective is loss reduction and equation (19) is used as the objective function when the objective is to reduce the overall power demand from the substation. The equation (19) is derived from Marti JR et al. [16], which considers the voltage dependent model of the loads as described by (16) and (17) and assumes small voltages. The system constraints are represented by following equations;

$$
\begin{aligned}
& V^{\min } \leq V_{i}^{r e} \leq V^{\max } \\
& I_{m, k}^{2} \leq I_{\max }^{2} \\
& \alpha_{i}=\sum_{t=h}^{T} \delta_{i, t} \\
& \delta_{i, t}=\left(\mu_{i, t}-\mu_{i, \mathrm{t}-\mathrm{h}}\right)^{2} \\
& \alpha_{i} \leq \alpha_{i}^{\max }
\end{aligned}
$$

$V^{\min }$ and $V^{\max }$ represent the minimum and the maximum nodal voltage limits. $I_{m k}^{2}$ and $I^{2}$ max is the actual and maximum current flowing through the branch $m, k \alpha_{i}$ is the total number of switching actions, $h$ is the fixed time step of the VVO operation and $\delta_{i, t}=1$ if change occurs in the status of unit $i$ from $t-h$ to $t$ and $\delta_{i, t}=0$ otherwise.

\begin{tabular}{|c|c|c|c|}
\hline Type & Water Heater & Space Heater & Refrigerator \\
\hline Tag & No. 1 & No. 2 & No. 3 \\
\hline Type & Electronics & Incand. Light & Flour. Light \\
\hline Tag & No. 4 & No. 5 & No. 6 \\
\hline Type & Dishwasher & Washing Machine & Clothes Dryer \\
\hline Tag & No. 7 & No. 8 & No. 9 \\
\hline Type & Electric Range & Vacuum Cleaner & Ventilation-Fan \\
\hline Tag & No. 10 & No. 11 & No. 12 \\
\hline Type & Induction Cooker & HP Sodium Light & Air Conditioner \\
\hline Tag & No. 13 & No. 14 & No. 15 \\
\hline
\end{tabular}

Table 1: List of typical appliance for residential area 
A capacitor bank is represented by an admittance element $B_{c}$ which appears in the corresponding diagonal element of $\mathrm{B}$

$$
B c=u i B c i
$$

Here $u i \in\{0,1\}$ represents the status of the capacitor bank connected at Node $i$. The tap changer capability is represented by equation (26) as explained in Ahmadi $\mathrm{H}$ et al. [8]

$$
a=\sum_{j=1}^{20} b_{j} x_{j}, \sum_{j=1}^{20} x_{j}=1
$$

$a$ is the transformer ratio, $x j \in\{0,1\}$ and $b j \in\{0.9,0.91, \ldots, 1.1\}$.

The 69-Node DS is used to perform simulations using GAMS platform and CIPLEX is used to solve the formulated optimization problem. The objective was first set to minimize the losses and to get clear understanding of the results, a continuous range of variation of the tap position (from 0.9 to 1.1 ) and capacitor sizes(0-1MVAr) is considered. The total losses as a function of tap position and reactive power injection are shown. The Figure 7 shows that, for losses to be minimum the required reactive power injection is $400 \mathrm{kVA}$. But injection of reactive power leads to increase in voltage level that in turn results in increasing the total demand by reverse CVR effect. The reactive power injection corresponding to minimum power demand is $166 \mathrm{kVA}$. Thus, the two objectives are conflicting in themselves.

Figure $8 \mathrm{~A}$ and $\mathrm{B}$ shows the simulation results as presented in the original paper.

Advantages: The paper presents very detailed modelling of the load and effect of transformer tap that enhances the accuracy of the model. The number of switching operation of the control devices has also been considered in the constraint set that makes the solution more practical. Furthermore, the linear model used to obtain the objective function makes the problem easier to solve even with standard solvers. The modelling technique used can be used as a base to carry out further research in this area. Although, the program is being based on load forecast can adopt to system changes and contingencies due to its fast response.

Drawbacks and scope for improvement: The formulated problem does not include restriction on the power factor range. Thus, it may increase the reactive power demand from the system.

The proposed mathematical model has different objectives for loss reduction and total power demand reduction. But, as explained in the previous section that both the objective functions are conflicting among themselves. Thus the proposed solution fails to integrate the loss reduction and the CVR into one optimization problem.

\section{Volt-Var control through joint optimization of capacitor bank switching renewable energy and home appliances}

General overview and contribution: This paper [9] is based on an ideal system in which each home has a Home Energy Management System(HEMS) and a cluster of such homes called as communities has Community Energy Management System (CEMS) and the various communities connected to the substation has its own Energy Management System (EMS). Each community member is assumed to possess various elastic loads like Electric Vehicles and renewable energy resources. The paper aims to minimize the cost of electric power delivered form the Figure 7. Total power loss at peak load substation. For the illustration purpose the system is shown in Figure 9. The mathematical model to determine the amount of Figure 9. Electricity transmission and distribution system power requirement and its cost in accordance with minimizing the losses is presented as;

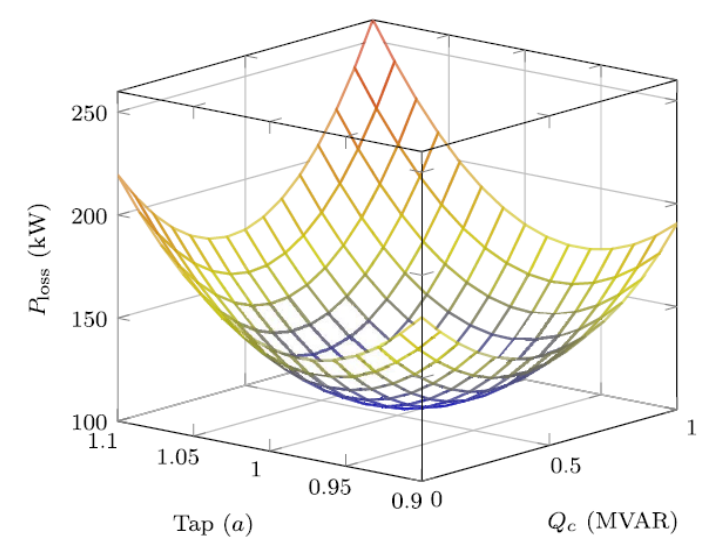

Figure 7: Total power loss at peak load

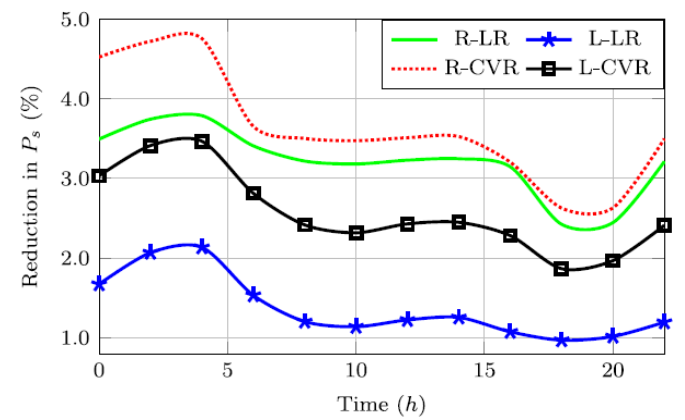

Figure 8A: Reduction in total active power demand.

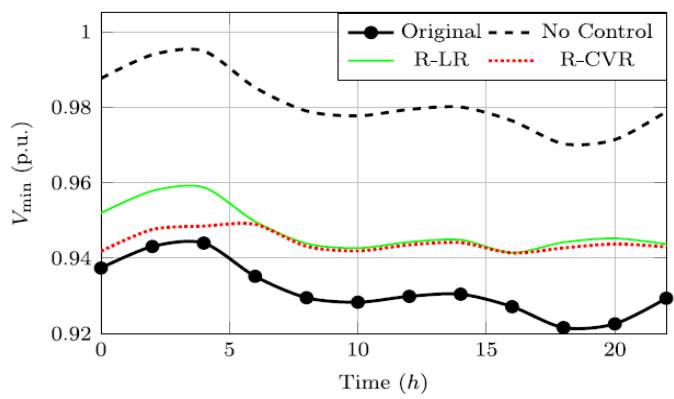

Figure 8B: Minimum nodal voltage, R-LR: loss reduction in Radial system, $\mathrm{R}$ CVR: results with CVR objective in radial system, original: all capacitors are off and $\mathrm{a}=1$ and no control: all capacitors are on and $\mathrm{a}=1.1$.

$$
\text { Objective : } \min \sum_{t=1}^{T} \mu\left(E_{g}^{t}\right)
$$

Here $\mu$ is the cost per unit of electricity and $E_{g}^{t}$ is the total amount of electricity which needs to be supplied at time $t$.

The objective function is subject to constraints of voltage drop, fixed minimum losses (close to zero), discrete capacitance of the capacitor banks, voltage constraints at each customer and the affect of tap changers as described in section III [9]. This optimization is performed on the basis of inputs received from the CEMS and the cost is sent back to the CEMS to further perform optimization to reduce the cost. The following objective function is used by each community to optimize the parameters to minimize the cost. 


$$
\text { Objective : } \min \sum_{t T} \pi_{t}\left(E_{i, j}^{t}+E_{i, j, e}^{t}-E_{i, j, s}^{t}\right)
$$

Where $\pi_{t}$ is the unit cost of the electricity at $t$ which is received from the EMS of the substation. $E_{i}^{t} \mathrm{j}, E_{i, j, s}^{t}$ and $E_{i, j e}^{t}$ are the total electricity consumption, the EV load and the total renewable energy of the community $\mathrm{r}_{\mathrm{i}, \mathrm{j}}$ at $t$. The objective function is subject to constraints of power consumption by each load, terminal voltage at each community, number of elastic loads on at a particular time slot, energy consumed or supplied by EV and energy consumed from renewables as described in section III [9].

Now if each community tries to minimize its cost then it will increase the power demand in off peak hours. If every community followed this strategy, then the overall effect would be conversion of off peak period into peak load period resulting in again increase in the cost per unit of energy. Thus, the author proposed a non-cooperative mixed strategy game between the communities which describes the best strategy to achieve Nash equilibrium is to minimize the deviation of the cost from the average cost. In this game, the community CEMS will act as the player of the game while the substation serves as the controller of the game. This is illustrated by Figure 10.

Advantages: The paper provides an effective framework for demand response integrating with optimal switching of control equipment to minimize the losses and energy cost as well as maintaining a flat load profile. The simulation results presented in the paper show the effectiveness of the system (cost saving up to 33\%) in the abovementioned aspects.

Drawbacks and scope for improvement: The proposed solution does make use of the opportunity to reducing the overall power demand by CVR and assumes a complete system visibility and complete control of loads using HEMS which makes it non-applicable in the present time.

\section{Non-linear VAR optimization using decomposition and coordination}

General overview and contribution: The paper [10] presents a fast method to solve the optimization problem of a large system by solving the problem for each sub systems as a non-linear program separately as shown in Figure 11. The subsystems are smaller and are easier to solve. The subsystems are selected based on sensitivity analysis. Each control device (OLTC or capacitor bank) is considered as a nucleus and sensitivity of buses are analysed with respect to the change in control variable of the nucleus.

$$
\left|\frac{\partial V_{i}}{\partial U_{\mathrm{K}}}\right| \geq \varepsilon_{\mathrm{K}} ; \varepsilon_{\mathrm{K}} \geq 0
$$

Where the term on the left-hand side of equation (29) represent the change in voltage of bus $i$ with the change in control variable of the control device at bus $\mathrm{k}$. The overall system problem is expressed as

Min

$L(x, u)$

Such that

$$
F(x, u)=0
$$

$G_{\min } \leq G(x, u) \leq G_{\max }$

First, the sub systems are selected based on voltage sensitivity criterion (29). Thus, the equation set (30) is divided into $\mathrm{n}$ sub optimization problems. Each subsystem is solved using a suitable NLP method and assuming the variables at the boundary buses (the last buses in a subsystem) to be fixed. After solution of each subsystem,

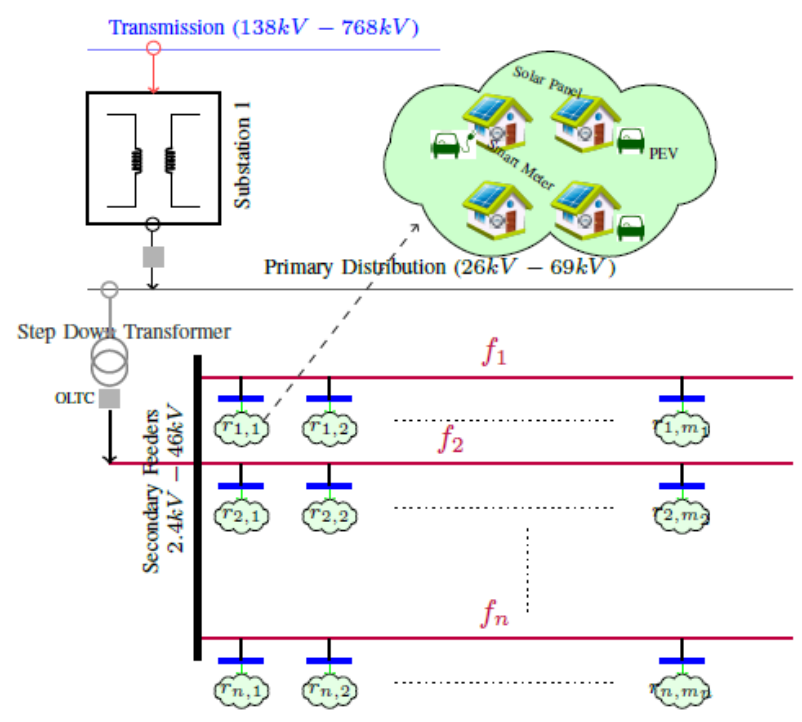

Figure 9: Electricity transmission and distribution system.

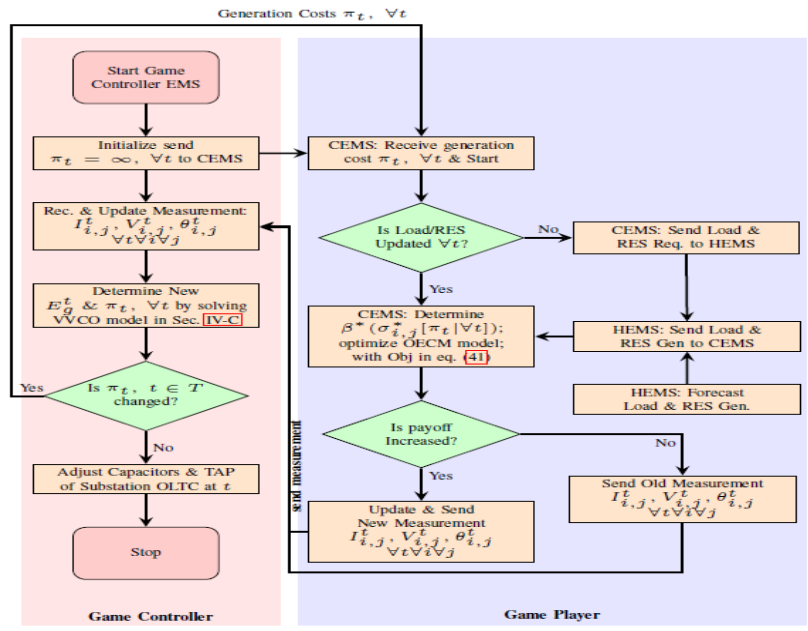

Figure 10: VVCO-ESM algorithm.

normal load flow is performed which acts as coordination function between the subsystems. The process is repeated till convergence.

Advantage: It is suitable for online applications and can act as guidance for the system operator to control reactive power and voltage. Linearized model is not used making the model accurate. It is applicable for any objective function and non-linear constraints.

Drawback and scope for improvement: The model is only applicable for single objective problems which limits its applicability on multi objective problems.

\section{Case Study}

The above discussed papers present a great opportunity to make our distribution system more efficient. These technologies have been used by many utilities across the world that proves its practical applicability. Hydro Quebec, BC Hydro, The Northwest Energy Efficiency Alliance, The Clinton Utilities board, Oklahoma Gas and Electric are among many agencies that have started VVO projects. The VVO project 
implemented by BC Hydro is discussed briefly as presented in Dabic $\mathrm{V}$ et al. $[17,18]$.

The first VVO pilot project was completed in 1996 with the objective of minimizing the distribution substation peak load demand and relieving transmission capacity constraints [17]. Load Tap Changers, fixed and switchable capacitors were installed to support the operation. The pilot project resulted in average (5 years) reduction of $1.1 \%$ reduction in peak load. Figure 12 illustrates the effect of voltage reduction on demand reduction for two consecutive days [17]. The thin yellow line represents the average bus voltage while thick yellow line represents consumed energy over these two days. VVO was active only on the second day and clear reduction is shown by overlapping the demands for the two days. In 2006, the objective was changed from demand reduction to energy conservation that resulted in energy savings of $1 \%$. On the basis of pilot project experiences, $\mathrm{BC}$ hydro has implemented VVO at three substation.

\section{Challenges faced}

i) As the Volt Var control is completely dependent on the system model, any changes in the system had to be updated regularly in the model.

ii) $\mathrm{VVO}$ at $\mathrm{BC}$ Hydro was executed real time every 15 minutes. But it may happen that at the time of execution, a voltage fluctuation happened, and the bus voltage deviated from the LTC set point. A mechanism had to be implemented in DMS to prevent issuance of the setpoint when the difference between estimated bus voltage and the setpoint is larger than pre-set deviation.

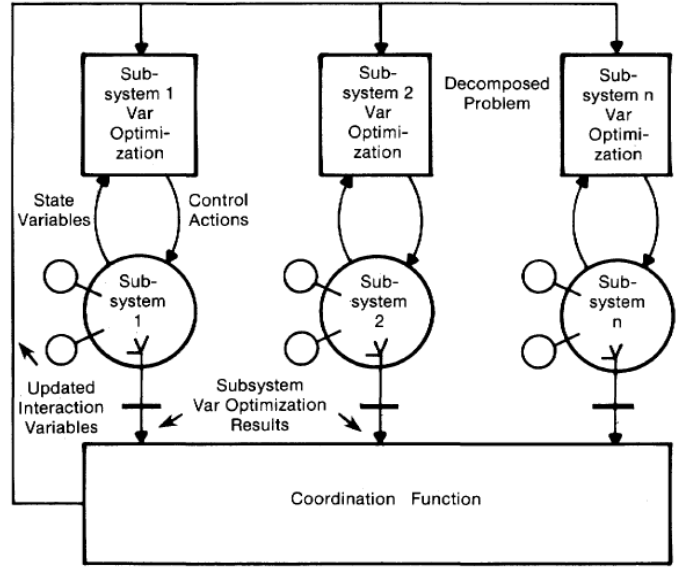

Figure 11: Strategy used for VVO method.

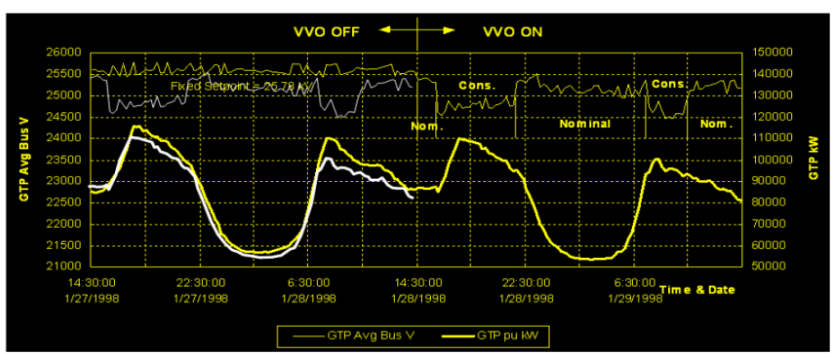

Figure 12: Achieved demand reduction by voltage reduction for two consecutive days. iii) The actual energy savings are daily compared with the estimated savings to detect a possible VVO execution problem.

iv) After voltage spikes from line switching, the LTC did not regulate the voltage to predefined set point. The problem is still under investigation.

v) The measurements obtained by the DMS were verified from the SCADA measurements to confirm the accuracy.

\section{Learnings}

i) During one of the tests, the LTC mechanical problem caused the tap to remain low and was undetected for a long time. Thus, complete system visibility is critical while implementing the system.

ii) The change in LTC design id required to include safety feature in case of loss of communication between the LTC and VVO.

iii) Due to the complexities involved, the project took a much longer time as compared to estimated time. Now their typical estimate time has been changed to 2 years.

iv) The load unbalance should be managed before the implementation of VVO for it to be more effective.

\section{Conclusion}

VVO technique applied nationwide in US would result in $3 \%$ of total energy savings [19]. But for such large-scale implementation, we need to develop concrete technological framework. There is a need to determine how to determine the communication technology that can best fit the deployment needs [20]. Furthermore, there is not a single study that integrates the concept of loss reduction and CVR into one optimized solution. These technical challenges can be overcome, if the utilities are committed to invest in this field. But today utilities are paid for the amount of energy they sell, and they have very less incentive to reduce the energy consumption. Presently, with many utilities implementing this technology and combined with their experience would certainly push more research in this filed and the drawbacks listed in this paper could be overcome soon.

\section{References}

1. National Electrical Manufacturers Association (NEMA) (2018, Mar 20). Volt Var Optimizatin Improves Grid Efficiency.

2. Sen PK, Lee KL (2014) Conservation voltage reduction technique: An application guideline for smarter grid. REPC

3. Arthur BK, Simonelli J (2013) Improvement of conservation voltage reduction energy savings via local voltage regulation. ECCE, pp: 2257-2263.

4. Electric Power Research Institute (2011) Grid Strategy 2011: Conservation Voltage Reduction and Volt VAR Optimization in the Smart Grid, California.

5. Singh S, Singh S (2016) A smart volt-var optimization engine for energy distribution system. ICETEESES.

6. Padilha-Feltrin A, Rodezno QD, Mantovani J (2015) Volt-VAR Multiobjective Optimization to Peak-Load Relief and Energy Efficiency in Distribution Networks. IEEE T Power Deliver 30: 618-626.

7. Bigovic MM, Radibratovic B, Lambert FC (2004) On Multiobjective Volt-VAR Optimization in Power Systems. In: Huwaii International Conference on System Science, Hawaii.

8. Ahmadi H, Marti J, Dommel H (2015) A Framework for Volt-VAR Optimization in Distribution Systems. IEEE Trans Smart Grid 6: 1473-1483.

9. Tushar MH, Assi C (2017) Volt-VAR Control through Joint Optimization of Capacitor Bank Switching, Renewable Energy, and Home Appliances. IEEE Trans Smart Grid, pp: 1-1. 
Citation: Mahendru A, Deshpande P (2018) A Review of Volt Var Optimization Techniques. J Electr Electron Syst 7: 265. doi: 10.4172/23320796.1000265

Page 8 of 8

10. Mansour MO, Abdel-rahman TM (1984) Non-Linear Var Optimization Using Decomposition And Coordination. IEEE Transactions on Power Apparatus and Systems PAS-103: 246-255.

11. Deb K, Pratap A, Agarwal S, Meyarivan TA (2002) fast and elitist multiobjective genetic algorithm: NSGA-II. IEEE Trans Evol Comput 6: 182-197.

12. IEEE task force on load representation for dynamic performance (1995). Bibliography on load models for power flow and dynamic performance simulation. IEEE T Power Syst 10: 523-538.

13. Deb K (2001) Multiobjective Optimization Using Evolutionary Algorithms. Chicester, UK, Wiley.

14. Singh R, Pal BC, Jabr RA (2010) Distribution system state estimation through Gaussian mixture model of the load as pseudo-measurement. IET Generation, Transmission \& Distribution 4: 50-59.
15. http://ewh.ieee.org/soc/pes/dsacom/testfeeders/index.html

16. Marti JR, Ahmadi H, Bashualdo L (2013) Linear power flow formulation based on a voltage-dependent load model. IEEE T Power Deliver 28: 1682-1690.

17. Dabic V, Siew C, Peralta J, Acebedo D (2010) BC Hydro's experience on Voltage VAR Optimization in distribution system. IEEE PES T\&D.

18. Dabic V, Atanackovic D (2015) Voltage VAR optimization real time closed loop deployment - BC Hydro challenges and opportunities. 2015 IEEE Power \& Energy Society General Meeting.

19. Scheider KP, Tuffner FK, Fuller JC, Singh R (2010) Pacific Northest National Laboratories, Evaluation of Conservation Volatge Reduction On National Level.

20. Alimisis V, Costa L, Tordjman P (2017) Voltage-VAr optimization (VVO) future trends, challenges and opportunities. ISGT. 